\title{
One More Assertion to Fermat's Last Theorem
}

\author{
Balasubramani Prema Rangasamy \\ Ramanujan Institute for Advanced Study in Mathematics, University of Madras, Chennai, India \\ Email: balguve@gmail.com
}

How to cite this paper: Rangasamy, B.P. (2020) One More Assertion to Fermat's Last Theorem. Advances in Pure Mathematics, 10, 359-369.

https://doi.org/10.4236/apm.2020.106022

Received: May 29, 2020

Accepted: June 27, 2020

Published: June 30, 2020

Copyright $\odot 2020$ by author(s) and Scientific Research Publishing Inc. This work is licensed under the Creative Commons Attribution International License (CC BY 4.0).

http://creativecommons.org/licenses/by/4.0/

\begin{abstract}
Around 1637, Fermat wrote his Last Theorem in the margin of his copy "It is impossible to separate a cube into two cubes, or a fourth power into two fourth powers, or in general, any power higher than the second, into two like powers". With $n, x, y, z \in \mathbf{N}$ (meaning that $n, x, y, z$ are all positive numbers) and $n>2$, the equation $x^{n}+y^{n}=z^{n}$ has no solutions. In this paper, I try to prove Fermat's statement by reverse order, which means no two cubes forms cube, no two fourth power forms a fourth power, or in general no two like powers forms a single like power greater than the two. I used roots, powers and radicals to assert Fermat's last theorem. Also I tried to generalize Fermat's conjecture for negative integers, with the help of radical equivalents of Pythagorean triplets and Euler's disproven conjecture.
\end{abstract}

\section{Keywords}

Fermat's Last Theorem, Fermat's Conjecture, Euler's Disproved Conjecture, Other Way of Taxi Cab Number and N-Tangled Object, Root of Prime Bases and Root of Integer Bases

\section{Introduction}

In our earlier classes, we have studied about factors, roots, powers, radicals and Pythagorean Theorem. Pythagorean Theorem says two squared numbers form a squared number. In other ways we can say, one certain number would be the root of the sum of two squared numbers. But this is not true for cubed and above numbers. Fermat thought like so. Around 1637, Fermat wrote his Last Theorem in the margin of his copy "It is impossible to separate a cube into two cubes, or a fourth power into two fourth powers, or in general, any power higher than the second, into two like powers". With $n, x, y, z \in \mathbf{N}$ (meaning that $n, x, y, z$ are all positive numbers) and $n>2$, the equation $x^{n}+y^{n}=z^{n}$ has no solutions. In 1769, Euler, by generalizing Fermat's Last Theorem, conjectured that "it is impossible 
to exhibit three fourth powers whose sum is a fourth power", "four fifth powers whose sum is a fifth power, and similarly for higher powers". The first counterexample to the conjecture, was found in 1966.

Over the next two centuries (1637-1839), the conjecture was proved for only the primes 3, 5, and 7, although Sophie Germain innovated and proved an approach that was relevant to an entire class of primes. In the mid-19th century, Ernst Kummer extended this and proved the theorem for all regular primes, leaving irregular primes to be analyzed individually. Separately, around 1955, Japanese mathematicians Goro Shimura and Yutaka Taniyama suspected a link might exist between elliptic curves and modular forms, two completely different areas of mathematics. Known at the time as the Taniyama-Shimura conjecture (eventually as the modularity theorem), it stood on its own, with no apparent connection to Fermat's Last Theorem. It was widely seen as significant and important in its own right, but was (like Fermat's theorem) widely considered completely inaccessible to proof. In 1984, Gerhard Frey noticed an apparent link between these two previously unrelated and unsolved problems. An outline suggesting this could be proved was given by Frey. The full proof that the two problems were closely linked was accomplished in 1986 by Ken Ribet, building on a partial proof by Jean-Pierre Serre, who proved all but one part known as the "epsilon conjecture". On hearing that Ribet had proven Frey's link to be correct, English mathematician Andrew Wiles, who had a childhood fascination with Fermat's Last Theorem and had a background of working with elliptic curves and related fields, decided to try to prove the Taniyama-Shimura conjecture as a way to prove Fermat's Last Theorem. In 1993, after six years of working secretly on the problem, Wiles succeeded in proving enough of the conjecture to prove Fermat's Last Theorem. Wiles's paper was massive in size and scope. A flaw was discovered in one part of his original paper during peer review and required a further year and collaboration with a past student, Richard Taylor, to resolve. As a result, the final proof in 1995 was accompanied by a smaller joint paper showing that the fixed steps were valid.

I think Fermat may not be had or known the usage of elliptical curves, Galois field, reducible and irreducibility. He conjectured that statement by what he known when he was alive. In 1994, Andrew wiles gave an assertion for Fermat's conjecture by using The Taniyama-Shimura-Weil conjecture, Modular theorem and elliptic curves. It may be different thing of Fermat's knowledge when he was stated that conjecture on 1637. Fermat generalized Pythagorean triplet. If he put $n=2$ in his conjecture, we would have no ambiguity to Pythagorean triplet. Euler also tried to generalize Pythagorean triplet but it disproven by $20^{\text {th }}$ century mathematicians. I too followed Euler's way before I found the quadruplet $1^{3}+6^{3}$ $+8^{3}=9^{3}$. In my previous paper titled "Some Extensions on Numbers" (advances in pure mathematics-published on Nov 2019). I tried to settle with Pythagoreantriplet, Fermat's last theorem and Euler's conjecture by prime numbers. With the help of following I tried to prove Fermat's last theorem. Such as [1]. L. J. Lander, T. R. Parkin, and John Selfridge-gave a counter example to Euler's 
sum of powers conjectures-AMS journal (1967)-pages (101-103), [2] Balasubramani Prema Rangasamy-Some Extensions on Numbers-Advances in Pure Mathematics-2019. pp. 944-958. [3], Wikipedia-gave some important introduction to know the extract of Fermat's last theorem and Euler's conjecture and [4] India-Tamil nadu SCERT high school mathematics books from $6^{\text {th }}$ standard to 10 standard gave ideas about rational, irrationals, radicals, powers and decimals.

In this paper, I try to prove Fermat's statement by reverse order, which means no two cubes forms cube, no two fourth power forms a fourth power, or in general no two like powers forms a single like power greater than the two. I used roots, powers and radicals to assert Fermat's last theorem. Also I tried to generalize Fermat's conjecture for negative integers, with the help of radical equivalents of Pythagorean triplets and Euler's disproven conjecture.

\section{Euler's Disproved Conjecture}

"It is impossible to exhibit three fourth powers whose sum is a fourth power", "four fifth powers whose sum is a fifth power, and similarly for higher powers". But

In 1967, L. J. Lander, T. R. Parkin, and John Selfridge conjectured that if

$$
\sum_{i=1}^{n} a_{i}^{k}=\sum_{j=1}^{m} b_{j}^{k},
$$

where $a_{i} \neq b_{j}$ are positive integers for all $1 \leq i \leq n$ and $1 \leq j \leq m$, then $m+n \geq k$.

Above conjecture disproved Euler's conjecture.

We can state above conjecture as,

Theorem 1: For any $t \in R$,

$$
\sum_{i=1}^{n} t a_{i}^{k}=\sum_{j=1}^{m} t b_{j}^{k},
$$

where $a_{i} \neq b_{j}$ are positive integers for all $1 \leq i \leq n$ and $1 \leq j \leq m$, then $m+n \geq k$.

Proof:

Let

$$
\sum_{i=1}^{n} a_{i}^{k}=\sum_{j=1}^{m} b_{j}^{k},
$$

where $a_{i} \neq b_{j}$ are positive integers for all $1 \leq i \leq n$ and $1 \leq j \leq m$, then $m+n \geq k$.

Then,

$$
a_{1}^{k}+a_{2}^{k}+a_{3}^{k}+\cdots+a_{n}^{k}=b_{1}^{k}+b_{2}^{k}+b_{3}^{k}+\cdots+b_{m}^{k}
$$

For any number $t$,

$$
\begin{aligned}
t a_{1}^{k}+t a_{2}^{k}+t a_{3}^{k}+\cdots+t a_{n}^{k} & =t b_{1}^{k}+t b_{2}^{k}+t b_{3}^{k}+\cdots+t b_{m}^{k} \\
t\left(a_{1}^{k}+a_{2}^{k}+a_{3}^{k}+\cdots+a_{n}^{k}\right) & =t\left(b_{1}^{k}+b_{2}^{k}+b_{3}^{k}+\cdots+b_{m}^{k}\right) \\
\sum_{i=1}^{n} t a_{i}^{k} & =\sum_{j=1}^{m} t b_{j}^{k}
\end{aligned}
$$


Corollary 1: If the sum of $k k^{\text {th }}$ powers of positive integers is itself a $k^{\text {th }}$ power $\sum_{i=1}^{k} a_{i}^{k}=b^{k}$. Also for any number, we can find $\sum_{i=1}^{k} t a_{i}^{k}=t b^{k}$.

Proof:

Let

$$
\sum_{i=1}^{n} a_{i}^{k}=\sum_{j=1}^{m} b_{j}^{k}
$$

where $a_{i} \neq b_{j}$ are positive integers for all $1 \leq i \leq n$ and $1 \leq j \leq m$, then $m+n \geq k$.

Then,

$$
a_{1}^{k}+a_{2}^{k}+a_{3}^{k}+\cdots+a_{n}^{k}=b_{1}^{k}+b_{2}^{k}+b_{3}^{k}+\cdots+b_{m}^{k}
$$

For any $n>(m=1)$

$$
a_{1}^{k}+a_{2}^{k}+a_{3}^{k}+\cdots+a_{n}^{k}=b_{1}^{k} \Rightarrow \sum_{i=1}^{k} a_{i}^{k}=b^{k}
$$

For any $t \in R$,

$$
\begin{gathered}
t a_{1}^{k}+t a_{2}^{k}+t a_{3}^{k}+\cdots+t a_{n}^{k}=t b^{k} \\
t\left(a_{1}^{k}+a_{2}^{k}+a_{3}^{k}+\cdots+a_{n}^{k}\right)=t b^{k} \\
\sum_{i=1}^{n} t a_{i}^{k}=t b^{k}
\end{gathered}
$$

Ex:

For any number $n$,

1) We can write Pythagorean triplets

$3^{2}+4^{2}=5^{2} ;$ As $6^{2}+8^{2}=10^{2} ; 9^{2}+12^{2}=15^{2} ; \cdots ;(3 n)^{2}+(4 n)^{2}=(5 n)^{2}$.

$5^{2}+12^{2}=13^{2} ;$ As $10^{2}+24^{2}=26^{2} ; 15^{2}+36^{2}=39^{2} ; \cdots$;

$(5 n)^{2}+(12 n)^{2}=(13 n)^{2}$.

2) Taxi cab number: $12^{3}+1^{3}=1729=10^{3}+9^{3}$

My way of taxi cab number: $1^{3}+6^{3}+8^{3}+10^{3}=1729$ and $1+8$ Plato's number $=1729$.

$$
(12 n)^{3}+n^{3}=1729 n^{3}=(10 n)^{3}+(9 n)^{3} .
$$

3) Plato's number: $3^{3}+4^{3}+5^{3}=6^{3}$

As $6^{3}+8^{3}+10^{3}=12^{3} ; 9^{3}+12^{3}+15^{3}=18^{3} ; \cdots ;(3 n)^{3}+(4 n)^{3}+(5 n)^{3}=(6 n)^{3}$.

One of my Triplet:

$$
1^{3}+6^{3}+8^{3}=1+216+512=729=9^{3}
$$

as $2^{3}+12^{3}+16^{3}=18^{3} ; 3^{3}+18^{3}+24^{3}=27^{3} ; \cdots ; n^{3}+(6 n)^{3}+(8 n)^{3}=(9 n)^{3}$.

Here $3^{3}+18^{3}+24^{3}=27^{3}$ is my number. Because one of the source triplet value is becoming resultant value.

i.e. $27+5832+13824=27^{3}$

4) $30^{4}+120^{4}+272^{4}+315^{4}=353^{4}$ as

$(30 n)^{4}+(120 n)^{4}+(272 n)^{4}+(315 n)^{4}=(353 n)^{4}$

5) $19^{5}+43^{5}+46^{5}+47^{5}+67^{5}=72^{5}$ as

$(19 n)^{5}+(43 n)^{5}+(46 n)^{5}+(47 n)^{5}+(67 n)^{5}=(72 n)^{5}$ 
For any Pythagorean triplets $a, b \& c$ we can say $c=\sqrt{a^{2}+b^{2}}$

Now we generalize above equation as

$$
c^{-1}=\sqrt[-2]{a^{2}+b^{2}}
$$

Definition 1: Let $a, k, n \in C$ then

$$
\sqrt[k]{a}=\sqrt[k n]{a^{n}}
$$

Definition 2: Let $a_{i}, b \in Z$, for any $k \in Z^{+}$such that $b=\sqrt[k]{\sum a_{i}^{k}}$.

Proof: Let $a_{1}^{k}, a_{2}^{k}, a_{3}^{k}, \cdots, a_{i}^{k} \in Z$ then from

L. J. Lander, T. R. Parkin, and John Selfridge conjecture

$$
\sum_{i=1}^{n} a_{i}^{k}=\sum_{j=1}^{m} b_{j}^{k}
$$

where $a_{i} \neq b_{j}$ are positive integers for all $1 \leq i \leq n$ and $1 \leq j \leq m$, then $m+n \geq k$.

We can say

$$
a_{1}^{k}+a_{2}^{k}+a_{3}^{k}+\cdots+a_{i}^{k}=b_{1}^{k}+b_{2}^{k}+b_{3}^{k}+\cdots+b_{j}^{k}
$$

For $m=1$,

$$
\begin{gathered}
a_{1}^{k}+a_{2}^{k}+a_{3}^{k}+\cdots+a_{i}^{k}=b_{1}^{k} \\
\sum_{i=1}^{n} a_{i}^{k}=b^{k} \\
\sqrt[k]{\sum_{i=1}^{n} a_{i}^{k}}=b
\end{gathered}
$$

Definition 3: Let $a_{i}, b \in Z$, for any $k \in Z$ such that

$$
b^{-1}=\sqrt[-k]{\sum a_{i}^{k}}
$$

Ex 1: let $a_{1}=30, a_{2}=120, a_{3}=272 \& a_{4}=315$ then

$$
a_{1}^{4}=810000, a_{2}^{4}=207360000, a_{3}^{4}=5473632256 \& a_{4}^{4}=9845600625 .
$$

Now,

$$
\begin{aligned}
a_{1}^{4}+a_{2}^{4}+a_{3}^{4}+a_{4}^{4}= & 810000+207360000+5473632256+9845600625 \\
= & 15527402881 \\
& 30^{4}+120^{4}+272^{4}+315^{4}=353^{4} \\
& \sqrt[4]{30^{4}+120^{4}+272^{4}+315^{4}}=353 .
\end{aligned}
$$

Let we see some example:
1) $30^{4}+120^{4}=810000+207360000=208170000=(120.117)^{4}$
2) $272^{4}+315^{4}=5473632256+9845600625=15319232881=(351.810)^{4}$
3) $353^{4}+30^{4}=15527402881+810000=15528212881=(353.004)^{4}$
4) $353^{4}+120^{4}=15527402881+207360000=15734762881=(354.172)^{4}$
5) $30^{4}+315^{4}=810000+9845600625=9846410625=(315.006)^{4}$.

\section{Facts}

1) Rational numbers $\times$ Rational numbers $=$ Rational numbers 
2) Rational numbers $\times$ Irrational numbers $=$ Irrational numbers

3) Irrational numbers $\times$ Rational numbers $=$ Irrational numbers

4) Irrational numbers $\times$ Irrational numbers $=$ may be Rational numbers.

5) Integer $\times$ integer $=$ Integer

6) Integer $\times$ decimal $=$ Integer or decimal

7) decimal $\times$ integer $=$ Integer or decimal

8) Decimal $\times$ decimal $=$ decimal.

\section{Prime Bases on Fermat's Last Theorem}

\section{Let we see following summations.}

Let $p_{i}$ are prime numbers then

$$
2+3+5+7+11+13=41
$$

For squared primes:

$$
\begin{gathered}
4+9=13=(3.6055 \cdots)^{2}=(3+0.6055 \cdots)^{2} ; \\
4+9+25=38=(6.1644 \cdots)^{2}=(5+1.1644 \cdots)^{2} ; \\
4+9+25+49=87=(9.3273 \cdots)^{2}=(7+2.3273 \cdots)^{2} ; \\
4+9+25+49+121=208=(14.4222 \cdots)^{2}=(11+3.4222 \cdots)^{2} ;
\end{gathered}
$$

For cubed primes:

$$
\begin{gathered}
8+27=35=(3.2710 \cdots)^{3}=(3+0.2710 \cdots)^{3} ; \\
8+27+125=160=(5.4288 \cdots)^{3}=(5+0.4288 \cdots)^{3} ; \\
8+27+125+343=503=(7.9528 \cdots)^{3}=(7+0.9528 \cdots)^{3} ; \\
8+27+125+343+1331=1724=(12.2405 \cdots)^{3}=(11+1.2405 \cdots)^{3} ;
\end{gathered}
$$

\section{For fourth exponent primes:}

$$
\begin{gathered}
16+81=97=(3.1382 \cdots)^{4}=(3+0.1382 \cdots)^{4} ; \\
16+81+625=722=(5.1836 \cdots)^{4}=(5+0.1836 \cdots)^{4} ; \\
16+81+625+2401=3123=(7.4755 \cdots)^{4}=(7+0.4755 \cdots)^{4} ; \\
16+81+625+2401+14641=17764=(11.5447 \cdots)^{4}=(11+0.5447 \cdots)^{4} ;
\end{gathered}
$$

By this way we concluded,

$$
2^{n}+3^{n}+5^{n}+7^{n}+11^{n}+13^{n}+\cdots+p_{i}^{n}=\left(p_{i}+B \cdot b_{1} b_{2} b_{3} b_{4} \cdots\right)^{n}
$$

where $B . b_{1} b_{2} b_{3} b_{4} \cdots \in R$.

From the above recursion, we formulate the result then we get,

$$
\sum_{i=1}^{k} p_{i}^{n}=\left(p_{i}+B \cdot b_{1} b_{2} b_{3} b_{4} \cdots\right)^{n}, \text { where } B \cdot b_{1} b_{2} b_{3} b_{4} \cdots \in R
$$

Preposition 1: Let $p_{i}$ are prime numbers then $\sum p_{i}^{n} \neq q^{n}$. Where $q$ is any prime. 


\section{Proof:}

Let $\sum p_{i}^{n}=P$ then

Case 1: If $P$ is prime, result is obvious.

Case 2: If $P$ is composite, we can write $P=s t+k$. if $k \neq 0$ then result is obvious.

Case 3: If $P$ is composite and $k=0$, then we can write $P=s t$. If $s, t$ are distinct primes then result is obvious. But if $s=t$ we get $\sum p_{i}^{n}=q^{n}$. This result contradict with (9). So $\sum p_{i}^{n} \neq q^{n}$. Where $q$ is any prime.

\section{Integer Bases on Fermat's Last Theorem}

\section{Let we see following summations of $\boldsymbol{n}^{\text {th }}$ exponent integers.}

Let $a_{i}$ are integers then

$$
1+2+3+4+5+6+7=21
$$

\section{For squared integers:}

$$
\begin{gathered}
2^{2}+3^{2}=4+9=13=(3.6055 \cdots)^{2}=(3+0.6055 \cdots)^{2} ; \\
5^{2}+9^{2}=25+81=106=(10.2956 \cdots)^{2}=(9+1.2956 \cdots)^{2} ; \\
25^{2}+79^{2}=625+6241=6866=(82.8613 \cdots)^{2}=(79+3.8613 \cdots)^{2} ; \\
35^{2}+959^{2}=1225+919681=920906=(959.6384 \cdots)^{2}=(959+0.6384 \cdots)^{2} ;
\end{gathered}
$$

\section{For cubed integers:}

$$
\begin{aligned}
2^{3}+3^{3}=8+27=35=(3.2710 \cdots)^{3}=(3+0.2710 \cdots)^{3} ; \\
15^{3}+49^{3}=3375+117649=121024=(49.4641 \cdots)^{3}=(49+0.4641 \cdots)^{3} ; \\
194^{3}+79^{2}=7301384+493039=7794423 \\
=(198.2719 \cdots)^{3}=(194+4.2749 \cdots)^{3} ; \\
335^{2}+7459^{2}=(7459.2252 \cdots)^{3}=(7459+0.2252 \cdots)^{3} ;
\end{aligned}
$$

\section{For fourth exponent integer:}

$$
\begin{gathered}
2^{4}+3^{4}=16+81=97=(3.1382 \cdots)^{4}=(3+0.1382 \cdots)^{4} \\
2^{4}+3^{4}+5^{4}=16+81+625=722=(5.1836 \cdots)^{4}=(5+0.1836 \cdots)^{4} \\
2^{4}+3^{4}+5^{4}+7^{4}=16+81+625+2401=3123=(7.4755 \cdots)^{4}=(7+0.4755 \cdots)^{4} \\
2^{4}+3^{4}+5^{4}+7^{4}+11^{4}=16+81+625+2401+14641=17764 \\
=(11.5447 \cdots)^{4}=(11+0.5447 \cdots)^{4} ; \\
26465^{4}+7895^{4}=(26517.2452 \cdots)^{4}=(26465+52.2452 \cdots)^{4}
\end{gathered}
$$

By this way we concluded,

$$
a_{1}^{n}+a_{2}^{n}+a_{3}^{n}+\cdots+a_{i}^{n}=\left(a_{i}+B \cdot b_{1} b_{2} b_{3} b_{4} \cdots\right)^{n}
$$

where $B . b_{1} b_{2} b_{3} b_{4} \cdots \in R$.

From the above recursion, we formulate the result then we get, 


$$
\sum_{i=1}^{k} a_{i}^{n}=\left(a_{i}+B \cdot b_{1} b_{2} b_{3} b_{4} \cdots\right)^{n} \text {. where } B \cdot b_{1} b_{2} b_{3} b_{4} \cdots \in R .
$$

From the above summations we concluded that, $n^{\text {th }}$ root of summation of $n^{\text {th }}$ exponent numbers is nearly greater than or equal to the biggest number of such $n^{\text {th }}$ exponent numbers.

\section{Irrationality of Numbers}

We know $\sqrt[n]{p}$ is always irrational, where $p$ is prime. So we can say $\sqrt[n]{2}$ is irrational and $\sqrt[n]{a^{k n}}$ is always rational, where $k, n \in Z$ and $a$ is rational.

Theorem 2: For any $k \in Z-\{-1,1\}, n \in Z$ and a number $a \in C-\{0,1\}$ we can say $\sqrt[n]{a^{k n}} \neq \sqrt[k n]{a^{n}}$

Proof:

Let $\sqrt[n]{a^{k n}} \neq \sqrt[k n]{a^{n}}$ then

$$
\left(a^{k n}\right)^{\frac{1}{n}} \neq\left(a^{n}\right)^{\frac{1}{k n}} \Rightarrow a^{\frac{k n}{n}} \neq a^{\frac{n}{k n}} \Rightarrow a^{k} \neq a^{\frac{1}{k}} .
$$

When we considering exponent,

Let $\sqrt[n]{a^{k n}}=\sqrt[k n]{a^{n}}$ then

$$
\left(a^{k n}\right)^{\frac{1}{n}}=\left(a^{n}\right)^{\frac{1}{k n}} \Rightarrow a^{\frac{k n}{n}}=a^{\frac{n}{k n}} \Rightarrow a^{k}=a^{\frac{1}{k}}
$$

This is true when $k=-1$ or 1

But this is contradict with $k \in Z-\{-1,1\}$. Hence $\sqrt[n]{a^{k n}} \neq \sqrt[k n]{a^{n}}$.

When we considering base,,

Let $\sqrt[n]{a^{k n}}=\sqrt[k n]{a^{n}}$ then

$$
\left(a^{k n}\right)^{\frac{1}{n}}=\left(a^{n}\right)^{\frac{1}{k n}} \Rightarrow a^{\frac{k n}{n}}=a^{\frac{n}{k n}} \Rightarrow a^{k}=a^{\frac{1}{k}}
$$

This is true when $a=0$ or 1 .

But this is contradict with $a \in C-\{0,1\}$. Hence

$$
\sqrt[n]{a^{k n}} \neq \sqrt[k n]{a^{n}}
$$

\section{Fermat's Last Theorem}

No three positive integers $a, b$, and $c$ satisfy the equation $a^{n}+b^{n}=c^{n}$ for any integer value $n>2$.

\section{Proof:}

Let $a, b \in Z^{+}$then for any positive integer $n>2 \mid n \in Z^{+}$such that $c^{n} \neq a^{n}+b^{n}$. Where $c \in Z^{+}$.

To prove above,

Let $c^{n}=a^{n}+b^{n}$ where $n>2 \mid a, b \& n \in Z^{+}$then we can say $c=\sqrt[n]{a^{n}+b^{n}}$. Now we check the possibilities for " $c$ " getting an integer value.

Case 1: Let $a=b$ then $a^{n}=b^{n}$. Now,

$$
\begin{aligned}
c & =\sqrt[n]{a^{n}+a^{n}}=\sqrt[n]{2 a^{n}} \\
& =a(\sqrt[n]{2}) \text { since } \sqrt[n]{a^{n}}=a \\
& =k \cdot d_{1} d_{2} d_{3} d_{4} d_{5} \cdots
\end{aligned}
$$


where $k$ is an integer and $d_{1} d_{2} d_{3} d_{4} d_{5}$ are decimal values.

Due to irrationality of $\sqrt[n]{2}$ we cannot get $\mathrm{c}$ is an integer.

Case 2: Let $a>b$ then $a^{n}>b^{n}$ and also we can say $a^{n}-h=b^{n}$ where $h$ is any integer.

Now,

$$
\begin{gathered}
c=\sqrt[n]{a^{n}+b^{n}}=\sqrt[n]{a^{n}+\left(a^{n}-h\right)}=\sqrt[n]{2 a^{n}-h} \\
=\sqrt[n]{2 a^{n}\left(1-\frac{h}{2 a^{n}}\right)}=a(\sqrt[n]{2})\left[\sqrt[n]{\left(1-\frac{h}{2 a^{n}}\right)}\right] \\
c=k \cdot d_{1} d_{2} d_{3} d_{4} d_{5} \cdots
\end{gathered}
$$

where $k$ is an integer and $d_{1} d_{2} d_{3} d_{4} d_{5}$ are decimal values. So we cannot get $c$ is an integer.

Case 3: Let $a<b$ then $a^{n}<b^{n}$ and also we can say $a^{n}=b^{n}-j$ where $j$ is any integer.

Now,

$$
\begin{gathered}
c=\sqrt[n]{a^{n}+b^{n}}=\sqrt[n]{\left(b^{n}-j\right)+b^{n}}=\sqrt[n]{2 b^{n}-j} \\
=\sqrt[n]{2 b^{n}\left(1-\frac{j}{2 b^{n}}\right)}=b(\sqrt[n]{2})\left[\sqrt[n]{\left(1-\frac{j}{2 b^{n}}\right)}\right] \\
c=k \cdot d_{1} d_{2} d_{3} d_{4} d_{5} \cdots
\end{gathered}
$$

where $k$ is an integer and $d_{1} d_{2} d_{3} d_{4} d_{5}$ are decimal values. So we cannot get $\mathrm{c}$ as an integer.

From the above cases we concluded that, ' $\mathcal{c}$ never gets integer value. "

Using above result we can state,

Theorem 3: No three integers $a, b$, and $c$ satisfy the equation $a^{n}+b^{n}=c^{n}$ for any positive integer $n>2$.

\section{Proof:}

Case 1: Let $a \in Z^{-}, b \in Z^{+}$and $|a|>b$, for any $n \in Z^{+ \text {Odd }}$, we get $a^{n}$ as negative integer. i.e. $(-a)^{n}=-a^{n}$. So we get $\left|-a^{n}\right|>b^{n} \quad$ Using case 2 , we get $-a^{n}+t=b^{n}$ where $t$ is an integer

Now,

$$
\begin{aligned}
c & =\sqrt[n]{-a^{n}+b^{n}}=\sqrt[n]{-a^{n}+\left(-a^{n}+t\right)}=\sqrt[n]{-2 a^{n}+t} \\
& =\sqrt[n]{2 a^{n}\left(\frac{t}{2 a^{n}}-1\right)}=a(\sqrt[n]{2})\left[\sqrt[n]{\left(\frac{t}{2 a^{n}}-1\right)}\right]=-k \cdot d_{1} d_{2} d_{3} d_{4} d_{5} \cdots
\end{aligned}
$$

where $k$ is an integer and $d_{1} d_{2} d_{3} d_{4} d_{5}$ are decimal values. So we cannot get $c$ as positive integer.

Case 2: Let $a \in Z^{+}, b \in Z^{-}$and $a<|b|$, for any $n \in Z^{+ \text {Odd }}$, we get $b^{n}$ as negative integer. i.e. $(-b)^{n}=-b^{n}$. So we get $a^{n}<\left|b^{n}\right|$ Using case 3 , we get $a^{n}=-b^{n}+v$ where $v$ is an integer

Now, 


$$
\begin{aligned}
c & =\sqrt[n]{a^{n}-b^{n}}=\sqrt[n]{\left(-b^{n}+v\right)-b^{n}}=\sqrt[n]{-2 b^{n}+v} \\
& =\sqrt[n]{2 b^{n}\left(\frac{v}{2 b^{n}}-1\right)}=b(\sqrt[n]{2})\left[\sqrt[n]{\left(\frac{v}{2 b^{n}}-1\right)}\right]=-k \cdot d_{1} d_{2} d_{3} d_{4} d_{5} \cdots
\end{aligned}
$$

where $k$ is an integer and $d_{1} d_{2} d_{3} d_{4} d_{5}$ are decimal values. So we cannot get $c$ as positive integer.

Case 3: Let $a, b \in Z^{-}$and let $-a=-b$, for any $n \in Z^{+ \text {Odd }}$, we get $\left|-a^{n}\right|=\left|-b^{n}\right|$ Using case 1 , Now,

$$
\begin{aligned}
c & =\sqrt[n]{-a^{n}-b^{n}}=\sqrt[n]{-2 a^{n}} \\
& =a(\sqrt[n]{-2}) \text { since } \sqrt[n]{a^{n}}=a \\
& =-k \cdot d_{1} d_{2} d_{3} d_{4} d_{5} \cdots
\end{aligned}
$$

where $k$ is an integer and $d_{1} d_{2} d_{3} d_{4} d_{5}$ are decimal values.

Due to irrationality of $\sqrt[n]{2}$ we cannot get $c$ is an integer.

Case 4: Let $a, b \in Z^{-}$and $-a>-b$, for any $n \in Z^{+ \text {Odd }}$, we get $-a^{n}>-b^{n}$ also we can say $-a^{n}=-b^{n}+x$ Where $x$ is any integer. Now,

$$
\begin{aligned}
c & =\sqrt[n]{-a^{n}-b^{n}}=\sqrt[n]{\left(-b^{n}+x\right)-b^{n}}=\sqrt[n]{-2 b^{n}+x} \\
& =\sqrt[n]{2 b^{n}\left(\frac{x}{2 b^{n}}-1\right)}=b(\sqrt[n]{2})\left[\sqrt[n]{\left(\frac{x}{2 b^{n}}-1\right)}\right]=-k \cdot d_{1} d_{2} d_{3} d_{4} d_{5} \cdots
\end{aligned}
$$

where $k$ is an integer and $d_{1} d_{2} d_{3} d_{4} d_{5}$ are decimal values. So we cannot get $c$ as positive integer.

Case 5: Let $a, b \in Z^{-}$and $-a<-b$, for any $n \in Z^{+ \text {Odd }}$, we get $-a^{n}<-b^{n}$ also we can say $-a^{n}+y=-b^{n}$ Where y is any integer. Now,

$$
\begin{aligned}
c & =\sqrt[n]{-a^{n}-b^{n}}=\sqrt[n]{-a^{n}+\left(-a^{n}+y\right)}=\sqrt[n]{-2 a^{n}+y} \\
& =\sqrt[n]{2 a^{n}\left(\frac{y}{2 a^{n}}-1\right)}=a(\sqrt[n]{2})\left[\sqrt[n]{\left(\frac{y}{2 a^{n}}-1\right)}\right]=-k \cdot d_{1} d_{2} d_{3} d_{4} d_{5} \cdots
\end{aligned}
$$

where $k$ is an integer and $d_{1} d_{2} d_{3} d_{4} d_{5}$ are decimal values. So we cannot get $c$ as a positive integer.

For any $n \in Z^{+ \text {Even }}$ in cases 1 to 5 , we get

1) Case 1 implies the result of case 2 of FLT proof.

2) Case 2 implies the result of case 3 of FLT proof.

3) Case 3 implies the result of case 1 of FLT proof.

4) Case 4 implies the result of case 3 of FLT proof.

5) Case 5 implies the result of case 2 of FLT proof. -

\section{Conclusions}

1) Due to irrationality of $\sqrt[n]{2}$ in $c=\sqrt[n]{a^{n}+b^{n}}$, we could not get $c$ as an integer. So Fermat's theorem is always true.

2) It is also true for negative integers.

3 ) Generally $n^{\text {th }}$ root of $2^{\text {nd }}$ sum of $n^{\text {th }}$ power of numbers is always non integer, 
where $n>2$.

\section{Conflicts of Interest}

The author declares no conflicts of interest regarding the publication of this paper.

\section{References}

[1] Lander, L.J. and Parkin, T.R. (1967) A Counter Example to Euler's Sum of Powers Conjectures. AMS Journal, 21, 101-103. https://doi.org/10.1090/S0025-5718-1967-0220669-3

[2] Rangasamy, B.P. (2019) Some Extensions on Numbers. Advances in Pure Mathematics, 9, 944-958. https://doi.org/10.4236/apm.2019.911047

[3] Wikipedia. Some Base Works for Fermat's Last Theorem and Euler's Conjecture.

[4] India-Tamil Nadu SCERT High School Mathematics Books. 